Tohoku J. Exp. Med., 2011, 224, 221-228

\title{
Exercise Improves Recovery after Ischemic Brain Injury by Inducing the Expression of Angiopoietin-1 and Tie-2 in Rats
}

\author{
Qingping Zheng, ${ }^{1}$ Danian Zhu, ${ }^{2}$ Yulong Bai, ${ }^{1}$ Yi Wu, ${ }^{1}$ Jie Jia ${ }^{1}$ and Yongshan $\mathrm{Hu}^{1,3}$ \\ ${ }^{1}$ Department of Rehabilitation, Huashan Hospital, Fudan University, Shanghai, P.R.China \\ ${ }^{2}$ Department of Physiology and Pathophysiology, Medical College of Fudan University, Shanghai, P.R.China \\ ${ }^{3}$ State Key Laboratory of Medical Neurobiology, Fudan University, Shanghai, P.R.China
}

\begin{abstract}
Post-ischemia angiogenesis plays a critical part in the recovery of neural networks. Angiopoietin (Ang) has received much attention recently due to its key role in neurovascular remodeling. Exercise is proved to contribute to angiogenesis in normal or injured human skeletal muscle. The therapeutic effect of exercise on central angiogenesis after cerebral ischemia, however, has not been studied. In the present study, we investigated the relationship between exercise and the expression of Ang-1, Ang-2, and Tie-2 receptor tyrosine kinase in the brain using a rat model of stroke, with right middle cerebral artery occluded (MCAO). Male Sprague-Dawley rats were randomly grouped $(n=12)$ : stroke-exercise (SE), stroke-no exercise (SNE) and sham-no exercise (SHAM). The SE group ran on a treadmill at a speed of $12 \mathrm{~m} / \mathrm{min}, 30 \mathrm{~min} /$ day for 2 weeks. Functional recovery was assessed with neurological evaluation scores. Brain infarction was measured by Nissl staining. Expression of Ang-1, Ang-2, and Tie-2 were compared by immunohistochemical and real-time PCR analyses. The infarct volume in the SE group was significantly reduced compared with the SNE group $(p<0.05)$. Ang-1 $(p<0.05)$ and Tie-2 $(p<0.05)$ and their mRNA expression $(p<0.01$ and $p<0.05$, respectively) were increased in SE animals at 2 weeks, whereas Ang-2 expression remained unchanged. In conclusion, enhanced expression of Ang-1 and Tie-2 by exercise improves recovery of brain function in MCAO rats. Our results suggest the importance of angiogenesis in rehabilitation for post-ischemia brain injury and help to explain the underlying mechanism.
\end{abstract}

Keywords: angiogenesis; angiopoietin-1; angiopoietin-2; exercise; middle cerebral artery occlusion Tohoku J. Exp. Med., 2011, 224 (3), 221-228. C 2011 Tohoku University Medical Press

Stroke is the leading cause of long-term disability worldwide. It is associated with serious neurological impairment and persistent physical deficits. Among the interventions for stroke rehabilitation, exercise has been shown to be beneficial for recovery of brain function, and is widely used. Physical exercise has been shown to improve motor function in stroke survivors (Macko et al. 2005; Luft et al. 2008). Positive results in animal studies have also been reported (Briones et al. 2005; Kim et al. 2005). Neuroprotection and recovery of brain structure induced by exercise have been documented and widely investigated, but the underlying mechanism remains unclear.

Neurovascular remodeling is a key component of recovery after stroke, in which angiogenesis within the brain may play a critical part (Arai et al. 2009). Current thinking supports the notion that angiogenesis promotes neurogenesis (Zhang et al. 2005) and that re-growth of vascular structures might provide the requisite molecular (as well as anatomic) support for recovering neural networks (Ohab et al. 2006).
Two angiogenic growth factors, vascular endothelial growth factor (VEGF) and angiopoietin (Ang), have key roles in the formation of new blood vessels (Zhang and Chopp 2002). These angiogenic growth factors might be used to establish stable, healthy and functional blood vessels in ischemic areas. In experimental systems, however, VEGF induces adverse effects such as edema (Bates et al. 2002), inflammation (Schoch et al. 2002), vascular leakage (Wang et al. 1996), hemorrhagic blood vessels, and even neurotoxicity (Manoonkitiwongsa et al. 2004).

The Ang family, including angiopoietin-1 (Ang-1) and Ang-2, carry out signaling through the receptor tyrosine kinase Tie-2. They exert their functions at later stages of vascular development (i.e., during vascular remodeling and maturation) (Davis et al. 1996; Suri et al. 1996) and have been known to increase neo-vascularization. Ang-1 is expressed on endothelial cells and in the choroid plexus (Dumont et al. 1994; Nourhaghighi et al. 2003), and has been shown to promote angiogenesis in the brain (Ward and Lamanna 2004). Ang-2 competitively inhibits the Ang-1/

Received February 8, 2011; revision accepted for publication June 10, 2011. doi: 10.1620/tjem.224.221

Correspondence: Yongshan Hu, M.D., Ph.D., Department of Rehabilitation, Huashan Hospital, Fudan University, 12 Middle Wulumuqi

Road, Shanghai 200040, P.R.China.

e-mail: drhuys@sina.com 
Tie-2 interaction, leading to disruption of blood vessels (Maisonpierre et al. 1997; Asahara et al. 1998). Furthermore, Ang-1 and Ang-2 protect the peripheral vasculature from leakage (Thurston et al. 1999; Zhu et al. 2005; Lee et al. 2009), which may account for its anti-edema effects after cerebral ischemia.

Physical exercise has been shown to cause morphologic and functional effects, including promoting angiogenesis in animals and humans (Kleim et al. 2002; Swain et al. 2003; Laughlin and Roseguini 2008). The therapeutic effects of peripheral activity on central angiogenesis after cerebral ischemia, however, have not been studied.

In the present study of ischemia/reperfusion in a rat model, we tested the hypothesis that physical exercise might promote expression of the Ang/Tie-2 pathway, and wondered if the changes correlated with reduced injury to the brain.

\section{Materials and Methods}

Ethical approval of the study protocol

This project was approved by the Animal Experimental Committee of Shanghai Fudan University (Shanghai, China). All experimental procedures were conducted in accordance with the guidelines of the National Institutes of Health Guide for the Care and Use of Laboratory Animals.

\section{Animals and groups}

Male Sprague-Dawley rats (age, 2.5 months; 220-250 g) were provided by Shanghai Laboratory Animal Center, Chinese Academy of Sciences (Shanghai, China). They were maintained under a 12-h light/dark cycle with food and water available ad libitum.

Rats were randomly grouped $(n=12)$ : stroke-exercise (SE), stroke-no exercise (SNE) and sham-no exercise (SHAM) before right middle cerebral artery occlusion (MCAO) for $1 \mathrm{~h}$. All rats went through three phases: familiarization with treadmill exercise; MCAO or MCAO-sham surgery; and treadmill intervention.

Outcome measures were monitored at baseline and at specific times throughout the 2-week post-injury period. This 2 -week period was felt to be sufficient to assure histological evidence of neuroplastic adaptations in our transient ( $1 \mathrm{~h})$ MCAO model because Stroemer et al. (1995) found synaptogenesis to become evident 14-60 days after permanent MCAO. Investigators undertaking the outcome-testing procedures were blinded to the group assignments.

\section{$M C A O$}

The MCAO procedure leading to focal cerebral ischemia was carried out as previously described (Longa et al. 1989). Surgical procedures were carried out under aseptic conditions in a Surgery Suite. Rats were anesthetized with $10 \%$ chloral hydrate $(300 \mathrm{mg} / \mathrm{kg}$, i.p. $)$. Body temperature was maintained at $37^{\circ} \mathrm{C}$ with a heat pad. The basic procedure consisted of blocking blood flow into the right MCA with an intraluminal 4-0 uncoated monofilament nylon suture (Ethilon, Johnson \& Johnson International, St Stevens Woluwe, Belgium) with a rounded tip introduced through the extracranial internal carotid artery (ICA). The suture was introduced $\sim 18-20 \mathrm{~mm}$ from the carotid bifurcation. With the origin of the MCAO, the endovascular suture remained in place for $1 \mathrm{~h}$. The suture was then retracted to allow reperfusion. The SHAM group underwent the same procedure but no arteries were ligated.

\section{Treadmill training}

The SE group underwent treadmill training that began $24 \mathrm{~h}$ after surgery. A motor-driven treadmill (Litai Biotechnology, Hangzhou, China) was used. Treadmill use was based on our previous study (Zheng et al. 2007). Rats were placed in the opposite direction to the movement of the belt (i.e., rats had to keep running to avoid an electric shock from the electrode). All rats had to run over a period of 3 days before the MCAO procedure. The treadmill training was $30 \mathrm{~min}$ per day, 5 days a week at $12 \mathrm{~m} / \mathrm{min}$ and $0^{\circ}$ slope over 2 weeks.

Rats in SNE and SHAM groups were maintained in the same living conditions as the rats in SE group, but treadmill training was not undertaken.

\section{Neurological evaluation}

Neurological evaluation was done 1-7 d, $11 \mathrm{~d}$ and $14 \mathrm{~d}$ after surgery. A neurologic grading system with a six-point scale (0-5) as described by Longa et al. (1989) was used: 5 = no apparent deficits; 4 $=$ left forelimb flexion; $3=$ decreased grip of the left forelimb while being pushed; 2 = left circling while tail is pulled; $1=$ spontaneous left circling; $0=$ no spontaneous movement. Rats with a score of 1-4 points were considered to be successful models and were included in the study.

\section{Assessment of infarct volume}

Two weeks after surgery, rats were anesthetized with chloral hydrate and killed by cardiac perfusion of saline followed by $4 \%$ paraformaldehyde in $0.1 \mathrm{M}$ phosphate buffer (PB), PH 7.4. Frozen coronal brain sections from ischemic rats were cut on a freezing microtome (Leica Microsystems, Nussloch GmbH, Heidelberg, Germany) at a thickness of $30 \mu \mathrm{m}$ from $2.0 \mathrm{~mm}$ to $-4.0 \mathrm{~mm}$ at the level of the bregma (from the frontoparietal (sensorimotor) cortex to the dorsolateral striatum). One brain slice was selected every adjacent 8 slices $(240 \mu \mathrm{m})$ to determine infarct volume in ischemic rats. Sections were stained with Nissl ( $1 \%$ toluidine blue O) to identify surviving cells. The infarct region in the MCA territory was defined as the area with reduced Nissl staining, or which displayed denaturation or necrosis of neurons (their nuclei becoming pyknotic and fragmented). Measurement was carried out using image analyzer software (Department of Physiology and Pathophysiology, Medical College, Fudan University, Shanghai, China). The total measured infarct volume for each brain was calculated by summation of the infracted area of all brain slices from the same hemisphere. Infarct volume was presented as a percentage of the volume of the contralateral hemisphere. To minimize the error introduced by edema and individual differences in measurement, an indirect method for calculating infarct volume (Swanson et al. 1990) was used.

\section{Immunohistochemical (IHC) staining}

Every eighth coronal section was used for IHC staining. Immunostaining for Ang-1, Ang-2 and Tie-2 was undertaken. Coronal brain sections were incubated first with antibodies (which were all from Boster Bio-engineering, Wuhan, China) against Ang-1 (rabbit polyclonal IgG antibody; 1:150), Ang-2 (rabbit polyclonal IgG antibody; 1:150) and Tie-2 (rabbit polyclonal IgG antibody; 1:200; Boster Bio-engineering) at $4{ }^{\circ} \mathrm{C}$ overnight respectively. They were followed by incubation with an avidin-biotin-peroxidase complex and development in $3^{\prime} 3^{\prime}$-diaminobenzidine tetrahydrochloride (DAB; 
Boster bio-engineering). Control experiments consisted of staining brain coronal tissue sections as outlined above but without primary antibodies.

\section{Quantitative analyses of immunolabeled areas \\ Measurements of positively stained cells (i.e., those with brown granules in their cytoplasm) were done on five coronal sections per rat. The total number of positively stained cells in the infarct (which lay in the frontoparietal cortices) and peri-infarct (which lay in the dorsolateral corpus striatum) regions on the ischemic side was counted under a microscope (Olympus BX5, Tokyo, Japan) with a $10 \times$ objective lens. The same procedure was undertaken in the SHAM group. All histological analyses were done in a blinded fash- ion.}

Quantitative real-time reverse transcription-polymerase chain reaction ( $q R T-P C R$ )

RNA was isolated at day 14 after MCAO using Trizol (Watson Biotechnologies, Shanghai, China). One-microgram of total RNA from each sample was reverse-transcribed to cDNA using a Mo-MLV First-strand Synthesis System (Promega, Madison, WI, USA) for a RT-PCR kit according to the manufacturer's protocol.

The primer sequences were as follows: Ang-1 forward primer, GCT GGC AGT ACA ATG ACA GT, and reverse primer, GTA TCT GGG CCA TCT CCG AC; Ang-2 forward primer, GAC CAG TGG GCA TCG CTA CG, and reverse primer, CTG GTT GGC TGA TGC TAC TG; Tie-2 forward primer, TGC CAC CAT CAC TCA ATA CC, and reverse primer, AAA CGC CAA TAG CAC GGT GA; and glyceraldehyde 3-phosphate dehydrogenase (GAPDH) forward primer, CTC TAA GGC TGT GGG CAA GGT CAT, and reverse primer, GAG ATC CAC CAC CCT GTT GCT GTA. SYBR Green RT-PCR amplification was carried out in a $25 \mu \mathrm{L}$ reaction volume that contained $12.5 \mu \mathrm{L}$ of SYBR Green Realtime PCR Master Mix (Toyobo, Osaka, Japan), $1 \mu \mathrm{L}$-each of the forward and reverse primer, and $2 \mu \mathrm{L}$ of diluted cDNA using the iCycler iQ ${ }^{\mathrm{TM}}$ Real-Time PCR Detection System (BioRad, Hercules, CA, USA). The thermal profile for SYBR Green RT-PCR was $95^{\circ} \mathrm{C}$ for $3 \mathrm{~min}$, followed by 45 cycles of $95^{\circ} \mathrm{C}$ for $30 \mathrm{~s}$, annealing temperature for $45 \mathrm{~s}$, and $72^{\circ} \mathrm{C}$ for $45 \mathrm{~s}$. A single amplification product was confirmed by running a melting curve for all PCRs.

\section{Statistical analyses}

Neurological deficit was expressed as a median value and was compared using the Kruskal-Wallis analysis. Values for other variables are mean \pm SEM. One-way factorial ANOVA analyses for SE, SNE, and SHAM groups were used to assess infarct volume, number of positively stained cells and mRNA expression of Ang-1, -2 and Tie-2, followed by post-hoc comparisons (with Dunnett's test) if required. A value of $p<0.05$ was considered significant. Statistical analyses were done using SPSS 18.0 software (SPSS, Chicago, IL, USA).

\section{Results}

Neurological evaluation

Changes in the results of neurological evaluation are shown in Table 1. The score of all rats before surgery was 5. After MCAO, rats were observed for neurological deficits: their neurological scores gradually increased. From the first day after MCAO, there was a significant difference in neurological evaluation scores among the three groups ( $p$ $=0.001)$. A subsequent analysis between two groups revealed that the neurological scores of SE and SNE groups were significantly $(p<0.017)$ lower than that of the SHAM group, and that they gradually improved with time. The improvement in neurological scores in the SE group occurred earlier than that in the SNE group. From day-3 onwards, the scores in the SE group were significantly higher than those in the SNE group, and this trend continued until the end of 2 weeks $(p<0.017)$.

\section{Infarct volume}

Nissl staining was performed to determine the extent of brain infarction at day14 after ischemia. Areas of damage were mainly in the cerebral cortex including the dorsolateral and lateral cortices, as well as in the lateral striatum (Fig. 1A) with reduced Nissl staining or containing dark pyknotic, necrotic cell bodies (Fig. 1B), while the neurons in the normal region (Fig. 1C) were orbicular-ovate with deep blue.

All brain slices were measured using an image analyzer (Image Q500W, Leica Microsystems, Milton Keynes, UK). The final infarct volume for each rat was expressed as mean \pm SEM (Fig. 2). At the end of 2 weeks, the infarct volume in the SE group $(4.00 \pm 2.00 \%)$ was significantly decreased compared with that in the SNE group $(19 \pm 9 \%)$ $(p<0.05)$. The SHAM group did not exhibit infarction.

\section{Overexpression of Ang after exercise}

At the end of 2 weeks, Ang- and Tie-2-positive cells were detected mainly in the infarct and peri-infarct regions on the ischemic side. Most of them were neuron-like, with round or oval bodies displaying long, slender projections (Fig. 3A). Some of them were like endothelial cells, and were equally distributed in both regions (Fig. 3B).

One-way ANOVA revealed a significant difference

Table 1. Neurological evaluation scores at different times after MCAO.

\begin{tabular}{lccccccccc}
\hline & $1 \mathrm{~d}$ & $2 \mathrm{~d}$ & $3 \mathrm{~d}$ & $4 \mathrm{~d}$ & $5 \mathrm{~d}$ & $6 \mathrm{~d}$ & $7 \mathrm{~d}$ & $11 \mathrm{~d}$ & $14 \mathrm{~d}$ \\
\hline SE & $2^{\#}$ & $2.5^{\#}$ & $3^{* \#}$ & $4^{* \#}$ & $4^{* \#}$ & $4^{* \#}$ & $4^{* \#}$ & $4^{* \#}$ & $4^{* \#}$ \\
SNE & $2^{\#}$ & $2^{\#}$ & $2^{\#}$ & $2^{\#}$ & $2^{\#}$ & $2.5^{\#}$ & $2.5^{\#}$ & $3^{\#}$ & $3^{\#}$ \\
SHAM & 5 & 5 & 5 & 5 & 5 & 5 & 5 & 5 & 5 \\
\hline
\end{tabular}

Neurological evaluation scores were presented as median values. SE, stroke-exercise group; SNE, stroke-no exercise group; SHAM, sham-no exercise group. ${ }^{*} p<0.017$ vs. SHAM group; ${ }^{*} p<0.017$ vs. SNE group. 


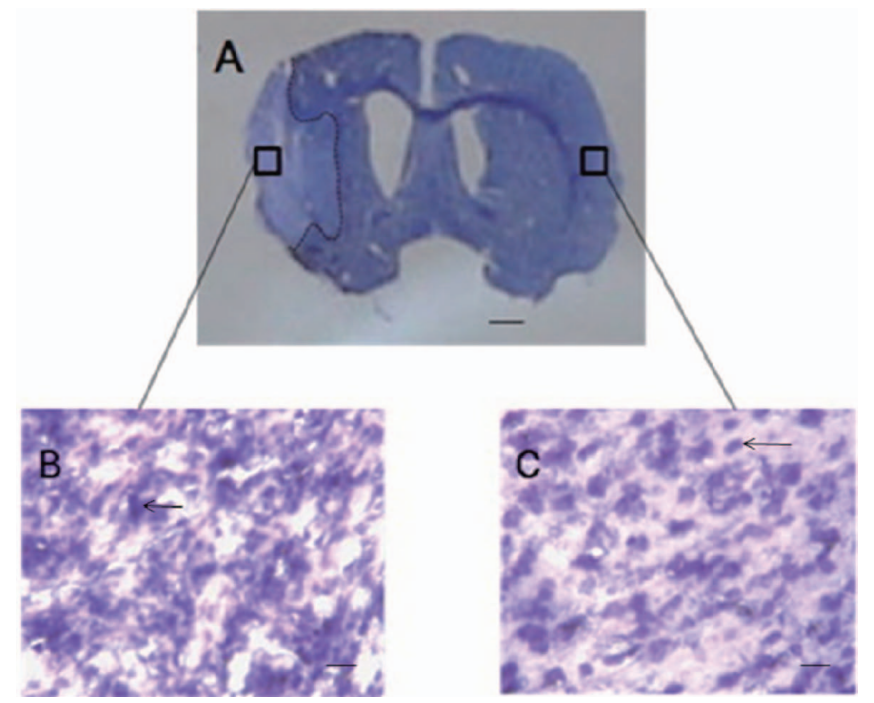

Fig. 1. Determination of infarct volume by Nissl staining 2 weeks after MCAO.

A: Section of rat brain, $30 \mu \mathrm{m}$-thick. The black squares indicate the infarct region. Scale bar $=1 \mathrm{~mm}$. B: Nissl staining of infarct cortex indicates denaturation and necrosis of neurons with pyknotic nuclei (arrow, $\times 400$ ). Scale bar $=25 \mu \mathrm{m}$. $\mathrm{C}$ : Nissl staining of the contralateral hemisphere shows homogeneous neurons with an orbicular-ovate nucleus (arrow, $\times$ 400). Scale bar $=25 \mu \mathrm{m}$.

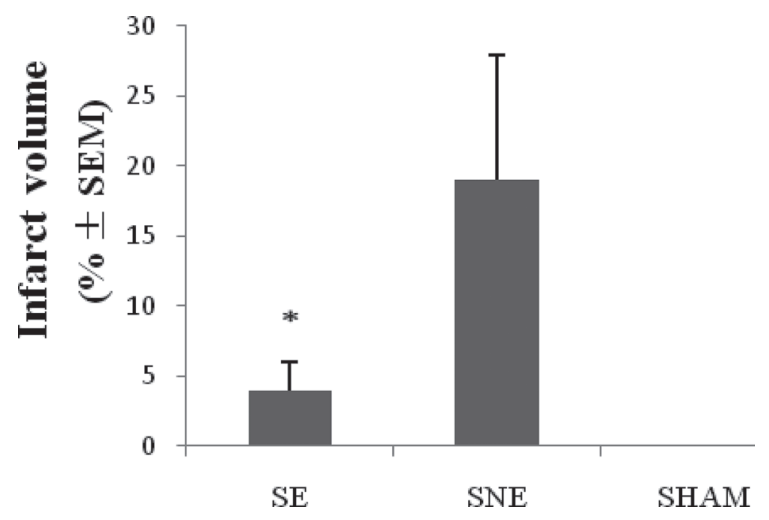

Fig. 2. Infarct volume in different groups 2 weeks after MCAO.

Infarct volume was mean \pm SEM. SE: stroke-exercise group; SNE: stroke-no exercise group; SHAM: sham-no exercise group.

${ }^{*} p<0.05$ vs. SNE group.

among groups in the expression of Ang and Tie-2 at the end of 2 weeks after MCAO. A subsequent post-hoc analysis between two groups revealed a significant difference between SHAM and SNE groups with regard to expression of Ang-1 and Tie-2 $(p<0.05)$ in the cortex or corpus striatum. Furthermore, in the cortex and corpus striatum, expression of Ang-1 and Tie-2 in SE was significantly increased as compared with the SNE group $(p<0.05)$. There was no significant difference in the expression of Ang-2 between the SNE and SHAM groups. Ang-2 expression increased in the SE group compared with that in the SNE group, but there was no significant difference between the two groups (Fig. 4).
Increased $m R N A$ expression in Ang after exercise

In the real-time RT-PCR reaction, the melting curves showed a sharply pointed peak with a uniform melting temperature, which ensured accuracy of the PCR products. According to the melting curve of the target genes and housekeeping gene, the peak value for Ang-1, Ang-2, Tie-2 and GAPDH were $89.5^{\circ} \mathrm{C}, 90^{\circ} \mathrm{C}, 87.5^{\circ} \mathrm{C}$, and $91^{\circ} \mathrm{C}$, respectively.

One-way ANOVA revealed a significant difference among groups in terms of the expression of Ang and Tie-2 at the end of 2 weeks after MCAO. Post-hoc analyses between two groups indicated significant increases in expression of Ang-1 $(p<0.01)$ and Tie-2 mRNA $(p<0.05)$ in the SE group compared with that in the SNE group. A significant increase in Tie-2 mRNA level was also found between the SNE and SHAM groups $(p<0.05)$. The expression of Ang-2 in the SE group was significantly increased as compared with that in the SHAM group $(p<$ 0.05 ) but, when compared with the SNE group, the increase in Ang-2 expression did not reach statistical significance (Fig. 5).

\section{Discussion}

The present study demonstrated that physical exercise can produce neuroprotective effects directly against ischemia/reperfusion injury in stroke. MCAO upregulated expression of Ang-1 and Tie-2, and physical exercise further increased their expression.

Some studies (Griesbach et al. 2004) indicated that final outcomes varied with the time of intervention and amount of exercise. An increased demand in oxygen induced by exercise can provoke compensatory mechanisms and metabolic stress. Excessive or premature exer- 

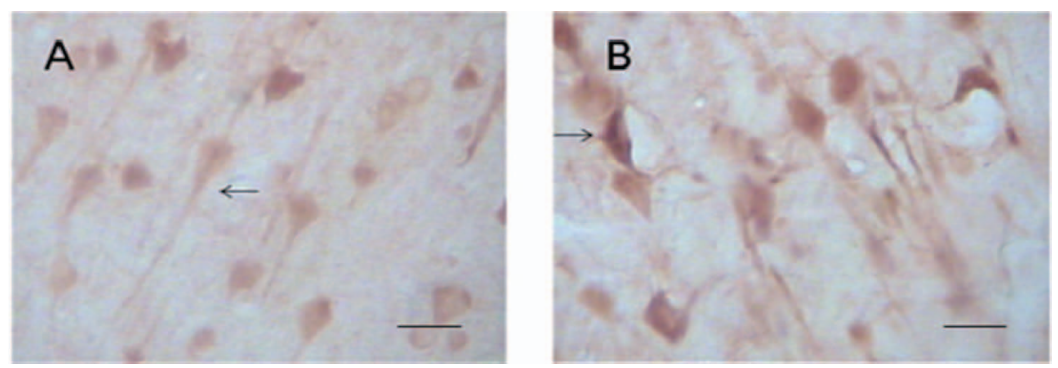

Fig. 3. Immunohistochemistry of the Ang/Tie-2 pathway after 2 weeks of exercise.

A: Ang-1-positively stained cells in the ischemic region; most are neuron-like (arrow, $\times 400$ ). B: Tie-2-positively stained cells in the ischemic region; some of them are like endothelial cells (arrow, $\times 400$ ). Scale bar $=25 \mu \mathrm{m}$.

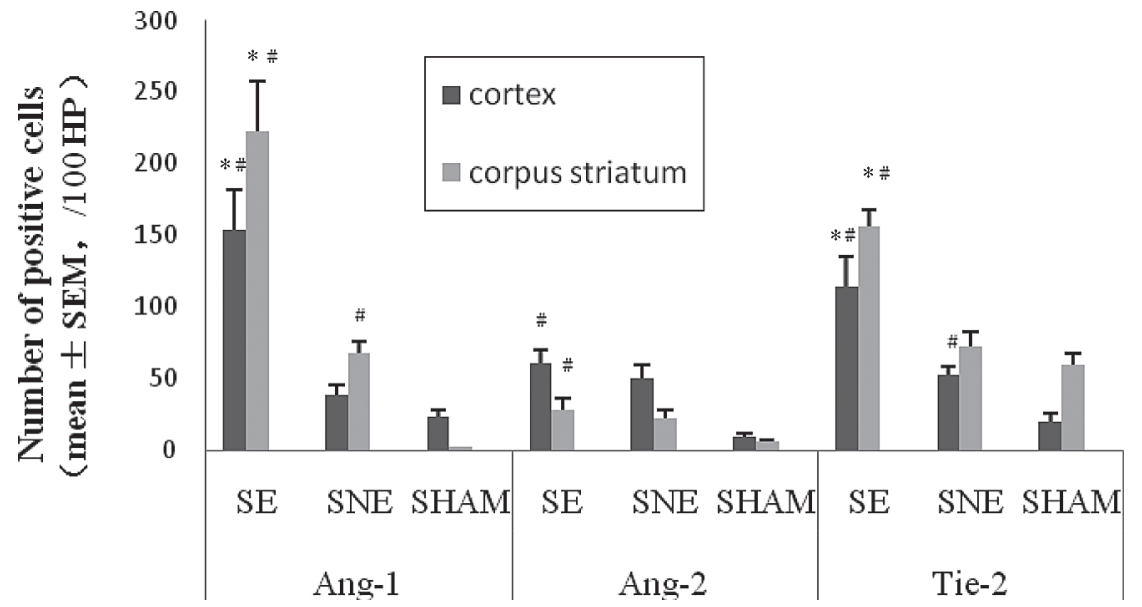

Fig. 4. Quantitative analyses by immunohistochemistry of Ang/Tie-2 pathway expression after 2 weeks of exercise. SE: stroke-exercise group; SNE: stroke-no exercise group; SHAM: sham-no exercise group. ${ }^{*} p<0.05$ vs. SNE group; ${ }^{*} p<0.05$ vs. SHAM group.

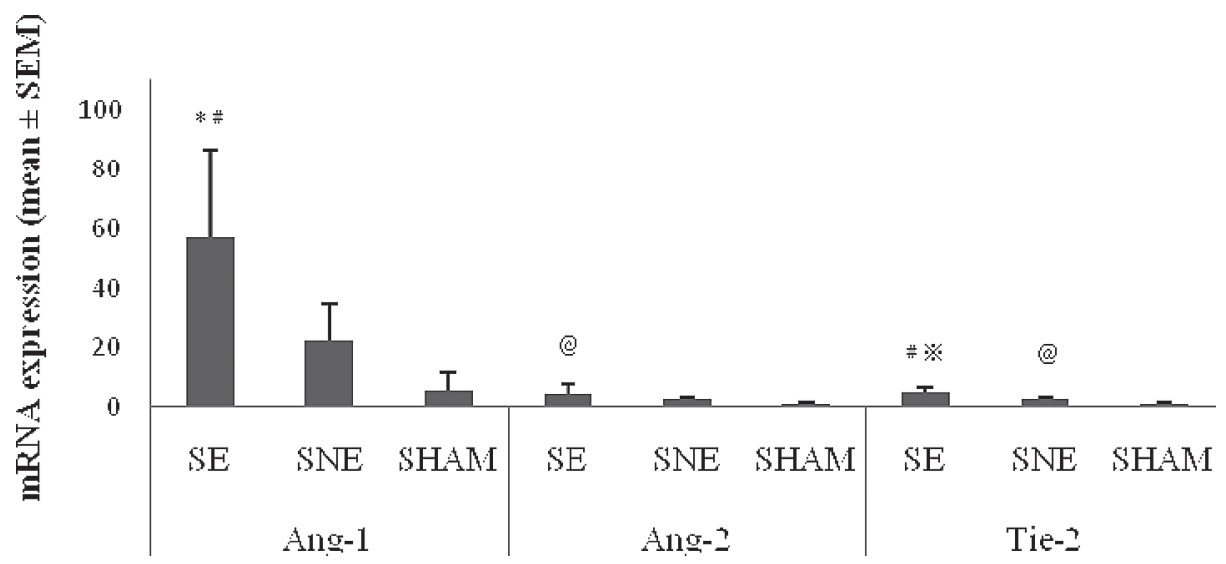

Fig. 5. mRNA expression of in the Ang/Tie-2 pathway after 2 weeks of exercise.

SE: stroke-exercise group; SNE: stroke-no exercise group; SHAM: sham-no exercise group.

${ }^{*} p<0.01$ vs. SNE group; ${ }^{\#} p<0.01$ vs. SHAM group; $* p<0.05$ vs. SNE group; ${ }^{\circledR} p<0.05$ vs. SHAM group.

cise may place a heavy burden on oxygen consumption and lead to poor outcomes. According to our previous rat model study (Zheng et al. 2007), treadmill training initiated $24 \mathrm{~h}$ after MCAO at $12 \mathrm{~m} / \mathrm{min}$ for $30 \mathrm{~min}$ a day proved to be an appropriate intervention for recovery of the ischemic brain. The present study indicated that 2 weeks of exercise resulted in significant reduction in the volume of ischemic lesions and improved functional outcome, which was consistent with previous studies.

It is well known that angiogenic factors have an essential role in regulating angiogenesis during embryonic development, normal growth of tissue, wound healing, the female reproductive cycle, and pathological processes (e.g., ischemia) (Carmeliet and Jain 2000; Yancopoulos et al. 2000). 
Angiogenesis in the brain occurs normally during early development (Ogunshola et al. 2000) and can occur under pathological conditions such as ischemia or hypoxia (Zhang and Chopp 2002). The role of angiogenesis in the recovery of stroke has received increasing attention in recent years, along with the development of new conceptual framework: the "neurovascular unit". Angiogenesis is associated with neurogenesis in the subventricular zone (SVZ) and subgranular zone (SGZ) (Alvarez-Buylla and Lim 2004; Wurmser et al. 2004). The Ang- $1 /$ Tie-2 pathway has been shown to promote migration of SVZ neuroblast cells after stroke (Cui et al. 2009) and to initiate survival responses in neural progenitor cells (NPCs) against cerebral ischemia and hypoxia (Bai et al. 2009). This ability of endogenous repair has already been used as cell therapy for brain ischemia (Shin et al. 2010; Zhao et al. 2010).

Exercise training is known to: profoundly change the morphology of different blood vessels along the arterial tree (Skalak et al. 1998); improve organ blood flow; and cause functional changes (Brown and Hudlicka 2003; Prior et al. 2004). Changes in cortical and striatal vasculature has also been detected after exercise training, and were thought to be attributed to persistent increases in neural activity (Kleim et al. 2002). However, information on such adaptations of cerebral vessels in stroke is scarce.

The present study showed a remarkable increase in the expression of Ang- 1 and Tie- 2 at the end of 2 weeks. This change comes not merely from the induction of ischemia but, more significantly, from physical exercise. This provides a possible mechanism of physical exercise in stroke, but the underlying mechanism needs to be clarified.

Exercise caused a robust and highly significant increase in the expression of Ang-1 and Tie-2, but the increase in Ang-2 expression was small and did not reach statistical significance. Ang-2, a natural antagonist of Ang1 , destabilizes the established vasculature, allowing formation of new vessels to occur in the endothelium (Maisonpierre et al. 1997; Yuan et al. 2009). Also, Ang-2 promotes or inhibits angiogenesis in different micro-environments (Thurston et al. 2000; Jones et al. 2001; Lobov et al. 2002; Zhu et al. 2005).

A recent study demonstrated that Ang-2 may also signal and facilitate endothelial inflammation (Fiedler and Augustin 2006; Parikh et al. 2006). Higher plasma levels of Ang-2 were associated with the risk of stroke recurrence in patients with lacunar infarction (Chen et al. 2010). Hence, the function of Ang-2 is quite complicated and remains incompletely characterized.

Angiogenesis is a very complex process that involves the activation, migration and proliferation of cells. Other than the Ang/Tie-2 pathway, there are many pro-angiogenic (e.g., fibroblast growth factor, transforming growth factor) and anti-angiogenic (e.g., endostatin, angiostatin) factors (Qutub et al. 2009). Exercise-induced endogenous neuroprotection in transient stroke could therefore probably also involve those mechanisms. Ultimately, this will lead to a coordinated expansion of various elements to support enhanced aerobic exercise.

Further studies are needed to provide direct evidence showing the causal relationship between exercise-induced motor improvement and cellular expression of angiogenesis. In addition, understanding how this is regulated in vivo by interaction with other growth factors is a continuing challenge.

In conclusion, treadmill exercise in rats subjected to transient MCAO reduced ischemia/reperfusion injury in stroke. The reduced damage to the brain was associated with angiogenesis and expression of angiogenic factors after exercise. The Ang/Tie-2 pathway triggered by physical exercise may act as one of the mechanisms of exercise-induced neuroprotection in stroke.

\section{Acknowledgments}

We thank Professor IZUMI Shinichi (Graduate School of Biomedical Engineering, Tohoku University) for comments on an earlier version of this manuscript.

\section{Conflict of Interest}

The authors declare no conflict of interest.

\section{References}

Alvarez-Buylla, A. \& Lim, D.A. (2004) For the long run: maintaining germinal niches in the adult brain. Neuron, 41, 683-686.

Arai, K., Jin, G., Navaratna, D. \& Lo, E.H. (2009) Brain angiogenesis in developmental and pathological processes: neurovascular injury and angiogenic recovery after stroke. FEBS J., 276, 4644-4652.

Asahara, T., Chen, D., Takahashi, T., Fujikawa, K., Kearney, M., Magner, M., Yancopoulos, G.D. \& Isner, J.M. (1998) Tie2 receptor ligands, angiopoietin-1 and angiopoietin-2, modulate VEGF-induced postnatal neovascularization. Circ. Res., 83, 233-240.

Bai, Y., Meng, Z., Cui, M., Zhang, X., Chen, F., Xiao, J., Shen, L. \& Zhang, Y. (2009) An Ang1-Tie2-PI3K axis in neural progenitor cells initiates survival responses against oxygen and glucose deprivation. Neuroscience, 160, 371-381.

Bates, D.O., Hillman, N.J., Williams, B., Neal, C.R. \& Pocock, T.M. (2002) Regulation of microvascular permeability by vascular endothelial growth factors. J. Anat., 200, 581-597.

Briones, T.L., Suh, E., Jozsa, L., Rogozinska, M., Woods, J. \& Wadowska, M. (2005) Changes in number of synapses and mitochondria in presynaptic terminals in the dentate gyrus following cerebral ischemia and rehabilitation training. Brain Res., 1003, 51-57.

Brown, M.D. \& Hudlicka, O. (2003) Modulation of physiological angiogenesis in skeletal muscle by mechanical forces: involvement of VEGF and metalloproteinases. Angiogenesis, 6, 1-14.

Carmeliet, P. \& Jain, R.K. (2000) Angiogenesis in cancer and other diseases. Nature, 407, 249-257.

Chen, J., Yu, H., Sun, K., Song, W., Bai, Y., Yang, T., Song, Y., Zhang, Y. \& Hui, R. (2010) Promoter variant of angiopoietin-2 and plasma angiopoietin-2 are associated with risk of stroke recurrence in lacunar infarct patients. Biochem. Biophys. Res. Commun., 398, 212-216.

Cui, X., Chen, J., Zacharek, A., Roberts, C., Yang, Y. \& Chopp, M. (2009) Nitric oxide donor up-regulation of SDF1/CXCR4 and Ang1/Tie2 promotes neuroblast cell migration after stroke. $J$. Neurosci. Res., 87, 86-95. 
Davis, S., Aldrich, T.H., Jones, P.F., Acheson, A., Compton, D.L., Jain, V., Ryan, T.E., Bruno, J., Radziejewski, C., Maisonpierre, P.C. \& Yancopoulos, G.D. (1996) Isolation of angiopoietin-1, a ligand for the TIE2 receptor, by secretion-trap expression cloning. Cell, 87, 1161-1169.

Dumont, D.J., Gradwohl, G., Fong, G.H., Puri, M.C., Gertsenstein, M., Auerbach, A. \& Breitman, M.L. (1994) Dominantnegative and targeted null mutations in the endothelial receptor tyrosine kinase, tek, reveal a critical role in vasculogenesis of the embryo. Genes Dev., 8, 1897-1909.

Fiedler, U. \& Augustin, H.G. (2006) Angiopoietins: a link between angiogenesis and inflammation. Trends Immunol., 27, 552-558.

Griesbach, G.S., Gomez-Pinilla, F. \& Hovda, D.A. (2004) The upregulation of plasticity-related proteins following TBI is disrupted with acute voluntary exercise. Brain Res., 1016, 154-162.

Jones, N., Iljin, K., Dumont, D.J. \& Alitalo, K. (2001) Tie receptors: new modulators of angiogenic and lymphangiogenic responses. Nat. Rev. Mol. Cell Biol., 2, 257-267.

Kim, M.W., Bang, M.S., Han, T.R., Ko, Y.J., Yoon, B.W., Kim, J.H., Kang, L.M., Lee, K.M. \& Kim M.H. (2005) Exercise increased BDNF and trkB in the contralateral hemisphere of the ischemic rat brain. Brain Res., 1052, 16-21.

Kleim, J.A., Cooper, N.R. \& VandenBerg, P.M. (2002) Exercise induces angiogenesis but does not alter movement representations within rat motor cortex. Brain Res., 934, 1-6.

Laughlin, M.H. \& Roseguini, B. (2008) Mechanisms for exercise training-induced increases in skeletal muscle blood flow capacity: differences with interval sprint training versus aerobic endurance training. J. Physiol. Pharmacol., 59 (suppl 7), 71-88.

Lee, S.W., Kim, W.J., Jun, H.O., Choi, Y.K. \& Kim, K.W. (2009) Angiopoietin-1 reduces vascular endothelial growth factorinduced brain endothelial permeability via upregulation of ZO-2. Int. J. Mol. Med., 23, 279-284.

Lobov, I.B., Brooks, P.C. \& Lang, R.A. (2002) Angiopoietin-2 displays VEGF-dependent modulation of capillary structure and endothelial cell survival in vivo. Proc. Natl. Acad. Sci. USA, 99, 11205-11210.

Longa, E.Z., Weinstein, P.R., Carlson, S. \& Cummins, R. (1989) Reversible middle cerebral artery occlusion without craniectomy in rats. Stroke, 20, 84-91.

Luft, A., Macko, R., Forrester, L., Goldberg, A. \& Hanley, D.F. (2008) Post-stroke exercise rehabilitation: what we know about retraining the motor system and how it may apply to retraining the heart. Cleve. Clin. J. Med., 75 (suppl 2), S83-86.

Macko, R.F., Ivey, F.M., Forrester, L.W., Hanley, D., Sorkin, J.D., Katzel, L.I., Silver, K.H. \& Goldberg, A.P. (2005) Treadmill exercise rehabilitation improves ambulatory function and cardiovascular fitness in patients with chronic stroke: a randomized, controlled trial. Stroke, 36, 2206-2211.

Maisonpierre, P.C., Suri, C., Jones, P.F., Bartunkova, S., Wiegand, S.J., Radziejewski, C., Compton, D., McClain, J., Aldrich, T.H., Papadopoulos, N., Daly, T.J., Davis, S., Sato, T.N. \& Yancopoulos, G.D. (1997) Angiopoietin-2, a natural antagonist for Tie2 that disrupts in vivo angiogenesis. Science, 277, $55-60$.

Manoonkitiwongsa, P.S., Schultz, R.L., McCreery, D.B., Whitter, E.F. \& Lyden, P.D. (2004) Neuroprotection of ischemic brain by vascular endothelial growth factor is critically dependent on proper dosage and may be compromised by angiogenesis. J. Cereb. Blood Flow Metab., 24, 693-702.

Nourhaghighi, N., Teichert-Kuliszewska, K., Davis, J., Stewart, D.J. \& Nag, S. (2003) Altered expression of angiopoietins during blood-brain barrier breakdown and angiogenesis. Lab. Invest., 83, 1211-1222.

Ogunshola, O.O., Stewart, W.B., Mihalcik, V., Solli, T., Madri, J.A.
\& Ment, L.R. (2000) Neuronal VEGF expression correlates with angiogenesis in postnatal developing rat brain. Brain Res. Dev. Brain Res., 119, 139-153.

Ohab, J.J., Fleming, S., Blesch, A. \& Carmichael, S.T. (2006) A neurovascular niche for neurogenesis after stroke. $J$. Neurosci., 26, 13007-13016.

Parikh, S.M., Mammoto, T., Schultz, A., Yuan, H.T., Christiani, D., Karumanchi, S.A. \& Sukhatme, V.P. (2006) Excess circulating angiopoietin-2 may contribute to pulmonary vascular leak in sepsis in humans. PLoS Med., 3, e46.

Prior, B.M., Yang, H.T. \& Terjung, R.L. (2004) What makes vessels grow with exercise training? J. Appl. Physiol., 97, $1119-1128$

Qutub, A.A., Mac, Gabhann, F., Karagiannis, E.D., Vempati, P. \& Popel, A.S. (2009) Multiscale models of angiogenesis. IEEE Eng. Med. Biol. Mag., 28, 14-31.

Schoch, H.J., Fischer, S. \& Marti, H.H. (2002) Hypoxia-induced vascular endothelial growth factor expression causes vascular leakage in the brain. Brain, 125, 2549-2557.

Shin, H.Y., Lee, Y.J., Kim, H.J., Park, C.K., Kim, J.H., Wang, K.C., Kim, D.G., Koh, G.Y. \& Paek, S.H. (2010) Protective role of COMP-Ang1 in ischemic rat brain. J. Neurosci. Res., 88, 1052-1063.

Skalak, T.C., Price, R.J. \& Zeller, P.J. (1998) Where do new arterioles come from? Mechanical forces and microvessel adaptation. Microcirculation, 5, 91-94.

Stroemer, R.P., Kent, T.A. \& Hulsebosch, C.E. (1995) Neocortical neural sprouting synaptogenesis, and behavioral recovery after neocortical infarction in rats. Stroke, 26, 2135-2144.

Suri, C., Jones, P.F., Patan, S., Bartunkova, S., Maisonpierre, P.C., Davis, S., Sato, T.N. \& Yancopoulos, G.D. (1996) Requisite role of angiopoietin-1, a ligand for the Tie2 receptor, during embryonic angiogenesis. Cell, 87, 1171-1180.

Swain, R.A., Harris, A.B., Wiener, E.C., Dutka, M.V., Morris, H.D., Theien, B.E., Konda, S., Engberg, K., Lauterbur, P.C. \& Greenough, W.T. (2003) Prolonged exercise induces angiogenesis and increases cerebral blood volume in primary motor cortex of the rat. Neuroscience, 117, 1037-1046.

Swanson, R.A., Morton, M.T., Tsao-Wu, G., Savalos, R.A., Davidson, C. \& Sharp, F.R. (1990) A semiautomated method for measuring brain infarct volume. J. Cereb. Blood Flow Metab., 10, 290-293.

Thurston, G., Rudge, J.S., Ioffe, E., Zhou, H., Ross, L., Croll, S.D., Glazer, N., Holash, J., McDonald, D.M. \& Yancopoulos, G.D. (2000) Angiopoietin-1 protects the adult vasculature against plasma leakage. Nat. Med., 6, 460-463.

Thurston, G., Suri, C., Smith, K., McClain, J., Sato, T.N., Yancopoulos, G.D. \& McDonald, D.M. (1999) Leakageresistant blood vessels in mice transgenically over-expressing angiopoietin-1. Science, 286, 2511-2514.

Wang, W., Merrill, M.J. \& Borchardt, R.T. (1996) Vascular endothelial growth factor affects permeability of brain microvessel endothelial cells in vitro. Am. J. Physiol., 271, C1973-1980.

Ward, N.L. \& Lamanna, J.C. (2004) The neurovascular unit and its growth factors: coordinated response in the vascular and nervous systems. Neurol. Res., 26, 870-883.

Wurmser, A.E., Palmer, T.D. \& Gage, F.H. (2004) Neuroscience. Cellular interactions in the stem cell niche. Science, 304, 1253-1255.

Yancopoulos, G.D., Davis, S., Gale, N.W., Rudge, J.S., Wiegand, S.J. \& Holash, J. (2000) Vascular-specific growth factors and blood vessel formation. Nature, 407, 242-248.

Yuan, H.T., Khankin, E.V., Karumanchi, S.A. \& Parikh, S.M. (2009) Angiopoietin 2 is a partial agonist/antagonist of Tie2 signaling in the endothelium. Mol. Cell. Biol., 29, 2011-2022.

Zhang, R.L., Zhang, Z.G. \& Chopp, M. (2005) Neurogenesis in the adult ischemic brain: generation, migration, survival and restorative therapy. Neuroscientist, 11, 408-416.

Zhang, Z. \& Chopp, M. (2002) Vascular endothelial growth factor 
and angiopoietins in focal cerebral ischemia. Trends Cardiovasc. Med., 12, 62-66.

Zhao, Y., Li, Z., Wang, R., Wei, J., Li, G. \& Zhao, H. (2010) Angiopoietin 1 counteracts vascular endothelial growth factorinduced blood-brain barrier permeability and alleviates ischemic injury in the early stages of transient focal cerebral ischemia in rats. Neurol. Res., 32, 748-755.

Zheng, Q.P., Hu, Y.S., Bai, Y.L., Sun, L.M., Zhu, D.N., Cui, X. \&
Jia, J. (2007) The study of the optimal time for exercise therapeutic intervention on MCAO rats. Fudan Univ. J. Med. Sci., 34, 895-898 (in Chinese).

Zhu, Y., Lee, C., Shen, F., Du, R., Young, W.L. \& Yang, G.Y. (2005) Angiopoietin-2 facilitates vascular endothelial growth factor-induced angiogenesis in the mature mouse brain. Stroke, 36, 1533-1537. 